EPJ Web of Conferences 66, 02041 (2014)

DOI: $10.1051 /$ epjconf/201466020 41

C Owned by the authors, published by EDP Sciences, 2014

\title{
Statistical gamma-ray emission of gold and its astrophysical implications
}

\author{
F. Giacoppo, a, F. Bello1, L.A. Bernstein ${ }^{2}$, D. Bleuel2, R.B. Firestone ${ }^{3}$, A. Görgen ${ }^{1}$, M. \\ Guttormsen $^{1}$, T. W. Hagen ${ }^{1}$, M. Klintefjord ${ }^{1}$, P.E. Koehler ${ }^{1}$, A.C. Larsen ${ }^{1}$, H. T. Nyhus ${ }^{1}$, T. \\ Renstrøm ${ }^{1}$, E. Sahin ${ }^{1}$, S. Siem ${ }^{1}$, and T. Tornyi ${ }^{1,4}$ \\ ${ }^{1}$ Department of Physics, University of Oslo, N-0316 Oslo, Norway \\ ${ }^{2}$ Lawrence Livermore National Laboratory, 7000 East Avenue, Livermore, CA 94550-9234, USA \\ ${ }^{3}$ Lawrence Berkeley National Laboratory, 1 Cyclotron Road, Berkeley, CA 94720-88R0192, USA \\ ${ }^{4}$ Institute of Nuclear Research, MTA ATOMKI, H-4026 Debrecen, Hungary
}

\begin{abstract}
The properties of the excited states of gold isotopes were investigated at the Oslo Cyclotron Laboratory. This study is important for the understanding of neutron capture rates in astrophysical plasmas relevant for heavy element nucleosynthesys.
\end{abstract}

\section{Introduction}

At the National Ingnition Facility (NIF, California, USA [1]) it is possible to study nuclear reactions in stellar-like plasma conditions. At NIF, a system of intense laser beams delivers up to $2 \mathrm{MJ}$ on a hydrogen target enclosed in a gas-filled gold hohlraum causing implosion of the fusion capsule. Then an intense neutron flux with a large sub-MeV component is produced. In this way one can reproduce the extreme temperature and density conditions in exploding stars. Recent measurements suggest that highly-excited quasi-continuum states in ${ }^{196} \mathrm{Au}$ and ${ }^{198} \mathrm{Au}$ formed via the ${ }^{197} \mathrm{Au}(\mathrm{n}, 2 \mathrm{n})$ and the ${ }^{197} \mathrm{Au}(\mathrm{n}, \gamma)$ reactions respectively on gold in the NIF hohlraum, might be interacting with the high energy density plasma (HEDP) in the hohlraum prior to emitting gammas. Indeed a modified population of the high-spin isomeric levels $\left(J^{\pi}=12^{-}\right)$with respect to the ground states $\left(J^{\pi}=2^{-}\right)$ in ${ }^{196,198} \mathrm{Au}$ has been measured than in absence of a plasma environment. If the nuclear plasma interaction (NPI) occurs before the nucleus thermalizes, i.e. the neutron capture process does not lead to the formation of an intermediary compound system, the ability of the nucleus to retain the captured neutron could change. Interactions of highly-excited states have never been considered before to evaluate neutron capture rates that are important inputs in large network calculations of $\mathrm{r}$ - and sprocess nucleosynthesis invoked to explain the origin of heavy elements. The NPI rates are in turn highly sensitive to nuclear level densities (NLDs) and gamma strength functions (GSFs). In this work we will present efforts to determine the NLD and GSF in ${ }^{196} \mathrm{Au}$ and ${ }^{198} \mathrm{Au}$ in order to inform calculations of NPIs on excited nuclear states.

\footnotetext{
a e-mail: francesca.giacoppo@fys.uio.no
} 

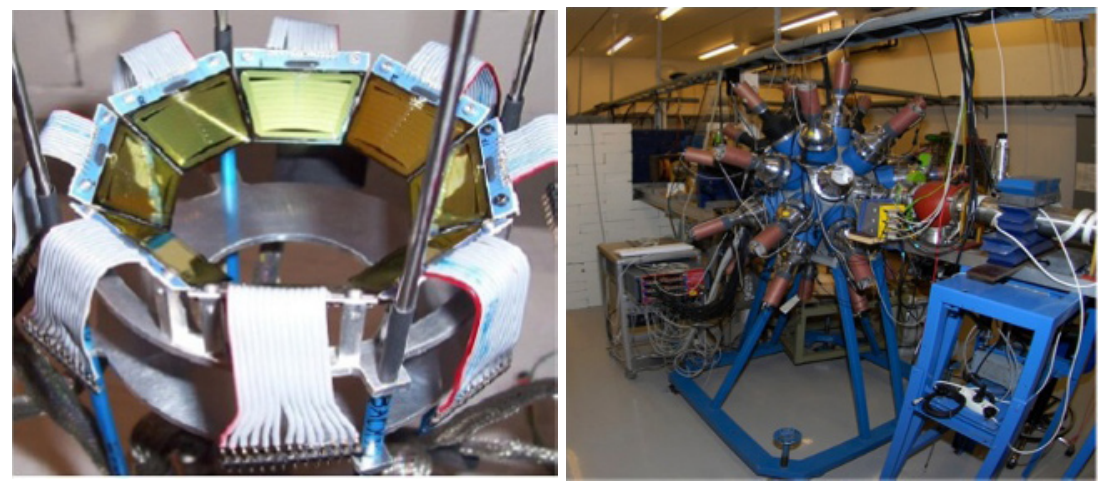

Figure 1: Left: the SiRi particle detector. Right: the $\gamma$-detector array CACTUS.

\section{Experimental setup and data analysis}

The nuclear physics group of the University of Oslo has developed a method to determine simultaneously the NLD and GSF from particle- $\gamma$ coincidence measurements [2,3]. Several experiments have been carried out at the Oslo Cyclotron Laboratory (OCL) using light particle beams on various target materials.

Recently, this method has been applied to measure the NLD and GSF of gold isotopes. The ${ }^{197} \mathrm{Au}(\mathrm{d}, \mathrm{p})$ and ${ }^{197} \mathrm{Au}\left({ }^{3} \mathrm{He}, \alpha\right)$ reactions were studied using self-supporting ${ }^{197} \mathrm{Au}$ targets with a thickness of $\sim 1.93 \mathrm{mg} / \mathrm{cm}^{2}$. The deuteron beam $(12.5 \mathrm{MeV})$ and ${ }^{3} \mathrm{He}$ beam $(34 \mathrm{MeV})$ were delivered by the MC-35 Scanditronix cyclotron. Particle- $\gamma$ coincidences were recorded in the Silicon Ring (SiRi) particle detector system [4] and the CACTUS multidetector system [5] shown in Fig. 1. The former consists of eight trapezoidal silicon $\Delta E-E$ telescopes mounted in a ring at $5 \mathrm{~cm}$ distance from the target in backward direction with respect of the beam, covering scattering angles in the range of $126^{\circ}$ - $140^{\circ}$, with a solid-angle coverage of $6 \%$ of $4 \pi$. Each telescope is made of a thin $(130 \mu m)$ front detector segmented into 8 strips and a $1550 \mu \mathrm{m}$ thick back detector with an angular resolution of $\Delta \theta=2^{\circ}$. By coupling the energy deposited into the $\Delta E$ detector with the $E$ detector the different types of charged particles are uniquely identified. From the reaction kinematics one can extract the excitation energy of the residual nucleus (with a resolution of $150-300 \mathrm{keV}$ ) from the energy and angle of the emitted particle. CACTUS is a spherical array of 28 collimated $5 " \times 5 " \mathrm{NaI}(\mathrm{Tl}) \gamma$-ray detectors sourrunding the target point. Its total detection efficiency is $15.2(1) \%$ for $\mathrm{E}_{\gamma}=1332 \mathrm{keV}$.

The spectrum of emitted $\gamma$-rays can then be analyzed for a given excitation energy of the residual nucleus. The $\gamma$-spectra are corrected for the detector response function through an unfolding procedure [6]. One of the main components of the Oslo method is an iterative subtraction technique developed to separate out the primary $\gamma$ transitions from the cascade of $\gamma$-rays originating from states at a given excitation energy [7]. The technique gives the $\mathrm{P}\left(\mathrm{E}, \mathrm{E}_{\gamma}\right)$ matrix, that expresses the probability of a $\gamma$-ray with energy $\mathrm{E}_{\gamma}$ decaying from a specific excitation energy $\mathrm{E}$ (see Fig. 2). The functional form of the NLD and the GSF can be derived from a simoultaneus fit of the P(E, $\left.E_{\gamma}\right)$ matrix. The two distributions have to be normalized using independent experimental data.

\section{Preliminary results}

Preliminary results for the NLD and the GSF of ${ }^{198} \mathrm{Au}$ are presented in Fig. 3. The NLD is normalized to fit the number of known discrete levels at low excitation energy and to the level density extracted 


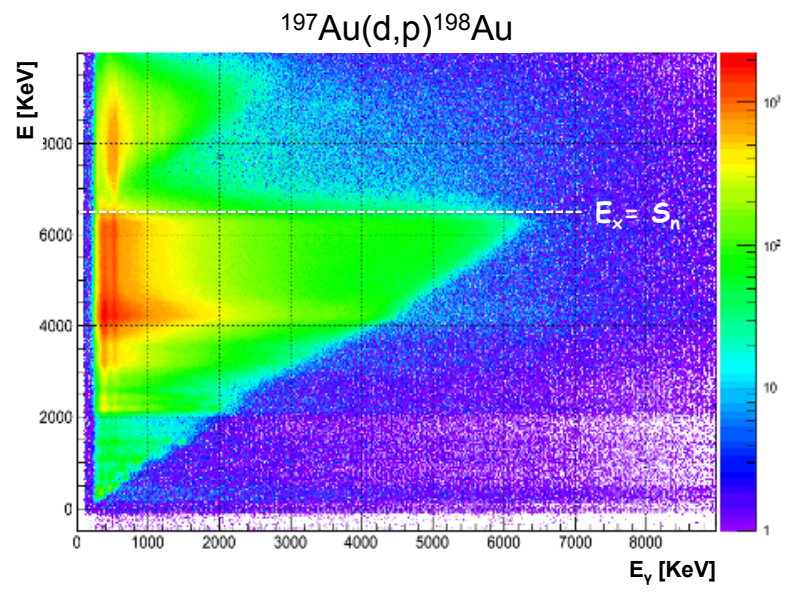

Figure 2. Matrix of $\gamma$-ray spectra tagged with the proton excitation energy from the ${ }^{197} \mathrm{Au}(\mathrm{d}, \mathrm{p})$ experiment at OCL in November 2012. The data used to extract the NLD and GSF correspond to the triangle below the neutron separation energy $S_{n}$.

from neutron resonance data at high excitation energy. In the latter case an interpolation is made to connect the value obtaioned at the neutron binding energy $\rho\left(\mathbf{S}_{n}\right)$ to our level density, based on the constant temperature (CT) model [8] :

$$
\rho(E)=\frac{1}{T} \exp \frac{E-E_{0}}{T}
$$

where the temperature $T$ charactherizes the slope and $E_{0}$ the shift in excitation energy of $\rho(E)$. The choice of a constant temperature extrapolation is well justified by the trend of the experimental level density data points which follows a straight line in a log-plot as shown in Fig. 3.

The normalized gamma strength function is compared in Fig. 3 with available experimental data at higher excitation energy: photoneutron cross-section data $\sigma\left(E_{\gamma}\right)$ [13-16] are converted to gamma strength $f\left(E_{\gamma}\right)$ through the relation:

$$
f\left(E_{\gamma}\right)=\frac{1}{3 \pi \hbar^{2} c^{2}}\left[\frac{\sigma\left(E_{\gamma}\right)}{E_{\gamma}}\right]
$$

The comparison with these data covering the region of the electric isovector giant dipole resonance (IVGDR) allows us to verify that our normalization is correct. The Oslo data clearly show an enhancement centered at $\sim 5.8 \mathrm{MeV}$ that will be referred to as the pygmy resonance. This result is in good agreement with the photoneutron data. The pygmy resonace in ${ }^{198} \mathrm{Au}$ was first oberved in 1967 by Bartholomew at al. and mentioned as an "anomalous bump" [9]. The nature of this collective mode is still controversial. In Fig. 3 the experimental gamma strength is also compared with theoretical models for the IVGDR resonance (generalized Lorentzian model GLO [10]) and for the magnetic spin-flip resonace (stantard Lorentzian model SLO [11]). In absence of an established theoretical prediction for the pygmy resonace a Gaussian distribution is chosen [12]. It is clear that additional terms are needed to fit the low-energy part of the experimental data. Also the region between $8.2 \mathrm{MeV}$ and 9.8 $\mathrm{MeV}$ shows an extra strength compared to the models.

\section{Summary and outlook}

The NLD and GSF of ${ }^{198}$ Au recently have been measured using the (d,p) reaction and the Oslo method. Normalization of the two functions is still prelimiary. A pigmy resonance-like enanchement has been confirmed at $\sim 5.8 \mathrm{MeV}$ in the gamma strength function. It is still not clear if the character of this collective mode is electric and/or magnetic. 

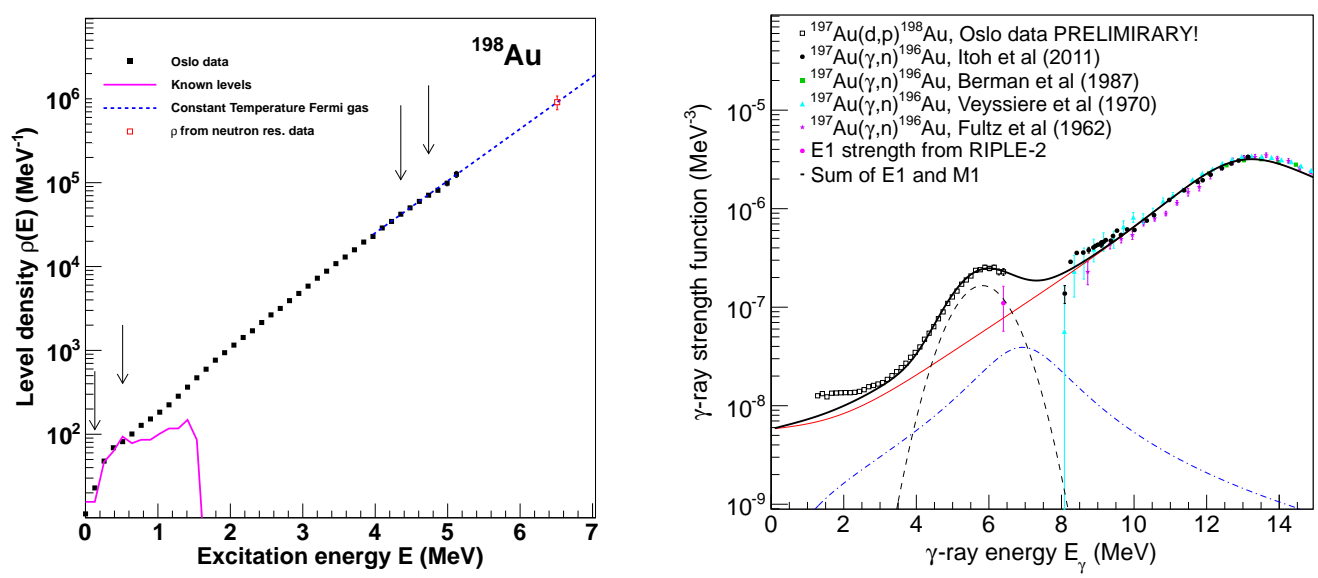

Figure 3: Left: Normalized nuclear level density of ${ }^{198} \mathrm{Au}$ (black points). The two sets of arrows define the area where the level density is normalized to the number of the discrete levels at low energy (violet line) and the level density at the neutron separation energy (red point). The dashed blue line is the costant temperature level density distribution. Right: Gamma-ray strength function of ${ }^{198} \mathrm{Au}$ from the recent ${ }^{197} \mathrm{Au}(\mathrm{d}, \mathrm{p})$ Oslo experiment together with previous results from photoneutron measurements [13-16]. Model parametrizations of the IVGDR (red line), the M1 spin-flip (dashed-point blue line) and the pygmy (dashed black line) resonances are summed up to reproduce the measured gamma strength. The models fail to reproduce the low gamma energy part $\left(\mathrm{E}_{\gamma}<2.5 \mathrm{MeV}\right)$ of the experimental data.

\section{References}

[1] G.H.Miller et al., Nucl. Fusion 44, S228 (2004)

[2] A. Schiller et al., Nucl. Instrum. Methods Phys. A 447, 498 (2000)

[3] A. C. Larsen et al., Phys. Rev. C 83, 034315 (2011)

[4] M. Guttormsen et al., Nucl. Instrum. Methods Phys. Res. A 648, 168 (2011)

[5] M. Guttormsen et al., Phys. Scr. T32, 54 (1990)

[6] M. Guttormsen et al., Nucl. Instrum. Methods Phys. Res. A 374, 371 (1996)

[7] M. Guttormsen et al., Nucl. Instrum. Methods Phys. Res., Sect. A 255, 518 (1987)

[8] A. Gilbert and A. G. W. Cameron, Can. Jour. Phys. 43, 1446 (1965)

[9] G. A. Bartholomew et al., Phys. Lett. 24B, 47 (1967)

[10] J. Kopecky and R. E. Chrien, Nucl. Phys. A 468, 285 (1987)

[11] T. Belgya et al., Handbook for Calculations of Nuclear Reaction Data, RIPL-2 (IAEA, Vienna, 2006). Available online at [http://www-nds.iaea.org/RIPL-2/]

[12] U. Agvaanluvsan et al., Phys. Rev. Lett. 102, 162504 (2009)

[13] O. Itoh et al., Jour. Nucl. Sc. Tech. 48, 1 (2011)

[14] B. L. Berman et al., Phys. Rev. C 36, 1286 (1987)

[15] A.Veyssiere et al., Nucl. Phys. A 159, 561 (1970)

[16] S. C. Fultz et al., Phys. Rev. 1271273 (1962) 